\title{
Multimodal imaging Gd-nanoparticles functionalized with Pittsburgh compound B or a nanobody for amyloid plaques targeting
}

\begin{abstract}
Aim: Gadolinium-based nanoparticles were functionalized with either the Pittsburgh compound B or a nanobody (B10AP) in order to create multimodal tools for an early diagnosis of amyloidoses. Materials \& methods: The ability of the functionalized nanoparticles to target amyloid fibrils made of $\beta$-amyloid peptide, amylin or Val30Metmutated transthyretin formed in vitro or from pathological tissues was investigated by a range of spectroscopic and biophysics techniques including fluorescence microscopy. Results: Nanoparticles functionalized by both probes efficiently interacted with the three types of amyloid fibrils, with $K_{D}$ values in 10 micromolar and 10 nanomolar range for, respectively, Pittsburgh compound B and B10AP nanoparticles. Moreover, they allowed the detection of amyloid deposits on pathological tissues. Conclusion: Such functionalized nanoparticles could represent promising flexible and multimodal imaging tools for the early diagnostic of amyloid diseases, in other words, Alzheimer's disease, Type 2 diabetes mellitus and the familial amyloidotic polyneuropathy.
\end{abstract}

First draft submitted: 10 March 2017; Accepted for publication: 24 May 2017; Published online: 21 June 2017

Keywords: amyloid imaging $\bullet$ amyloidoses $\bullet$ gadolinium-based nanoparticles $\bullet$ nanobody - $\mathrm{PIB}$

Amyloidoses are a set of human diseases in which a normally soluble peptide or protein aggregates into insoluble and highly organized aggregates referred to as amyloid fibres. These aggregates are rich in cross- $\beta$-sheet structures $[1,2]$. So far, 36 proteins are associated with either local (e.g., Type 2 diabetes mellitus [T2DM] and Alzheimer's disease [AD]) or systemic (e.g., familial polyneuropathy and myeloma) amyloid depositions [3]. These diseases inflict enormous psychosociological and economic burdens. For example, AD and T2DM affect, respectively, approximately 44 and 415 million people worldwide $[4,5]$.

Symptoms of amyloidoses, especially in the cases of systemic amyloidoses, can be very subtle and vary widely from person to person and also depend on which organs are affected. The diagnosis of amyloidosis and especially the early diagnosis, remains therefore a great challenge and often requires several invasive examinations [6,7].

Therefore, there is a need to develop sensitive and minimally invasive tools to detect amyloid deposits, in order not only to establish the diagnosis of amyloidosis [8] but also allow to follow-up the efficiency of treatment by monitoring the evolution of the amyloid load [9]. Nowadays, a number of imaging techniques can be used for these purposes; they include PET and MRI to detect the deposition of $A \beta$ amyloid in the brain of $A D$ patients [10,11], scintigraphy to detect V30MTTR fibrils involved in cardiac amyloidosis [12], autoradiography to target islet amyloid polypeptide (IAPP) fibrils in T2DM [13], etc. However, each of these techniques presents allows obtaining an outstanding spatial resosome limitations. For example, while MRI
Jonathan Pansieri ${ }^{\ddagger}, 1$, Marie Plissonneau ${ }^{\ddagger 2,3}$, Nathalie Stransky-Heilkron ${ }^{4}$, Mireille Dumoulin ${ }^{5}$, Laurence Heinrich-Balard ${ }^{6}$, Pascaline Rivory ${ }^{6}$, Jean-François Morfin7, Eva Toth7, Maria Joao Saraiva ${ }^{8}$, Eric Allémann ${ }^{4}$, Olivier Tillement ${ }^{3}$, Vincent Forge ${ }^{1}$, François Lux ${ }^{\ddagger, 3}$ \& Christel Marquette ${ }^{*, \neq, 1}$ 'Laboratoire de Chimie et Biologie des Métaux, Université Grenoble Alpes, CEA Life Sciences Division, CNRS, 17 rue des Martyrs, 38054 Grenoble Cedex 9, France ${ }^{2}$ Nano-H S.A.S, 38070 Saint Quentin Fallavier, France

${ }^{3}$ Institut Lumière Matière, University of Lyon, University of Claude Bernard Lyon 1, CNRS, F-69622, Lyon, France ${ }^{4}$ School of Pharmaceutical Sciences, University of Geneva, University of Lausanne Pharmaceutical technology, Quai Ernest-Ansermet 30, 1211 Geneva, Switzerland

${ }^{5}$ Laboratory of Enzymology \& Protein Folding, Centre for Protein Engineering, InBioS, University of Liege Sart Tilman, 4000 Liege, Belgium

${ }^{6}$ University of Lyon, University of Claude Bernard Lyon 1, ISPB Faculté de Pharmacie, MATEIS UMR CNRS 5510, 69373 Lyon, France.

${ }^{7}$ Centre de Biophysique Moléculaire, CNRS, UPR 4301, Université d'Orléans, Rue Charles Sadron, 45071 Orléans, France ${ }^{8}$ Instituto de Inovação e Investigação em Saúde (I3S), University of Porto, Portugal; Molecular Neurobiology Group, IBMC - Institute for Molecular \& Cell Biology, University of Porto, 4150-180 Porto, Portugal

*Author for correspondence: christel.marquette@cea.fr

${ }^{\ddagger}$ Authors contributed equally

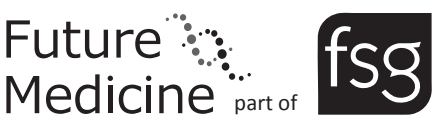


lution it strongly lacks sensitivity (1-0.01 millimolar range) [14]. On the other hand, PET offers an excellent sensitivity (10-1 pM range) but has a low spatial resolution [15]. In order to overcome these limitations, the development of probes allowing multimodal imaging approaches (i.e., combination of PET and MRI) will represent a breakthrough in the diagnosis of amyloidoses $[16,17]$. In this context, nanoparticles have emerged in the past decades as a very promising tool to enhance the contrast and the sensitivity of these different imaging techniques.

In this work, we have functionalized gadolinium (Gd) based multimodal nanoparticles (NPs), so called AGuIX with specific probes for the diagnosis of amyloidoses. AGuIX are made of a polysiloxane network surrounded by several DOTAGA $\left(\mathrm{Gd}^{3+}\right)$ chelates $[18,19]$. AGuIX NPs have a diameter smaller than $5 \mathrm{~nm}$ and have been already efficiently used for MRI and radiosensitization [20]. Free ligands are available at the surface of AGuIX and can be used to chelate radioisotopes (e.g., ${ }^{68} \mathrm{Ga}^{3+}$ or ${ }^{111} \mathrm{In}^{3+}$ ), in order to perform PET or single-photon emission computed tomography imaging [14,21]. Moreover, the covalent grafting of a near infrared dye, such as cyanine5.5 (Cy5.5), is also achievable for optical imaging [22]. Finally, we have recently shown that AGuIX can be functionalized with specific $A \beta$ peptides to selectively target and image $A \beta$-amyloid fibrils [23]. However, while the results obtained clearly demonstrate that these functionalized NPs constitute a valuable multimodal imaging tool to selectively target $A \beta$ fibrils, they displayed a low affinity $(100 \mu \mathrm{M}$ range) for their target. Therefore, the aim of the present work is to functionalize AGuIX with molecules having higher affinity for amyloid fibrils, in order to develop a sensitive tool for early diagnosis of amyloidoses.

Among the molecules binding to amyloid fibrils with relatively high affinity, thioflavin (ThS or ThT) is probably the most used probe for the detection of amyloid aggregates, both in vitro for biochemical characterization and ex vivo for histological studies [24]. Several thioflavin-derived molecules have been designed to further increase its affinity for amyloid fibrils in order to enable PET applications [11]. One of these molecules, the Pittsburgh compound B (PIB), shows an affinity in the nanomolar range toward $\beta$-amyloid deposits $[11,25-$ 26] and successfully targets senile plaques in Alzheimer's patient [27]. This compound can be coupled to metal ion such as $\mathrm{Gd}^{3+}$ for MRI or to radioisotopes such as ${ }^{111} \mathrm{In}^{3+}$ for single-photon emission computed tomography [28] or ${ }^{11} \mathrm{C}$ or ${ }^{18} \mathrm{~F}$ for PET. As consequence, PIB is considered as the clinical 'gold standard' probe for amyloidoses including AD.

Another approach to image amyloid fibrils consists of functionalizing NPs with antibodies or antibody fragments displaying a high specificity and affinity for the targeted fibrils [29]. For example, an enhanced amyloid- $\beta$ plaques detection by MRI was observed within AD transgenic mouse using SuperParamagnetic Iron Oxide Nanoparticles functionalized with an antiA $\beta P P$ antibody [30]. In this work, we have chosen to functionalize AGuIX with the B10AP which is a chimeric protein made of B10, a nanobody (i.e., the variable domain of heavy-chain antibodies) and Escherichia coli alkaline phosphatase (AP). B10 has been selected by phage display from a synthetic library of nanobodies using $A \beta_{(1-40)}$ fibrils as bait [31]. It also recognizes amyloid fibrils of several proteins (made in vitro and on tissues) including insulin, glucagon, $\beta 2$-microglobulin, serum amyloid A protein and immunoglobulin light chain (AL) [32]. AP forms homodimers and therefore B10AP exhibits two copies of B10 within one molecule of the chimera; such a bivalent construct exhibits a higher overall affinity for $A \beta_{(1-42)}$ amyloid fibrils with a lower dissociation constant $\left(K_{\mathrm{D}}: 7 \mathrm{nM}\right.$ and $475 \mathrm{nM}$ for B10AP and B10, respectively) [32]. Moreover, the phosphatase activity of AP allows an easy and straightforward monitoring of B10AP binding to the amyloid fibrils.

In this work, we have used PIB and B10AP as grafting ligands on AGuIX NPs, in order to perform active targeting on $A \beta_{(1-42)}$ fibrils which deposit in the brain of AD patients; IAPP fibrils that accumulate in Langerhans Islet of patients affected with T2DM; and V30M-TTR fibrils associated with familial amyloidotic polyneuropathy (FAP).

\section{Methods}

NPs synthesis

AGuIX were synthesized as previously described $[18,19]$ (Nano-H SAS, Saint-Quentin Fallavier, France). These NPs are composed of a polysiloxane network surrounded by covalently grafted DOTAGA $(1,4,7,10$-tetraazacyclododecane-1-glutaric anhydride-4,7,10-triacetic acid, CheMatech, Dijon, France) and $\mathrm{Gd}^{3+}$ chelates, with 5 or $7 \%$ free DOTA. NPs concentrations are stated in mol. $\mathrm{L}^{-1}$ of Gd element.

\section{Functionalization of AGuIX NP with PIB NP}

PIB derivative was synthesized as previously described [33]. In order to functionalize AGuIX with PIB, a polyethylene glycol bis-carboxymethyl (PEG) was first grafted on NP as previously described [23]; after purification, the AGuIX@PEG were freezedried. Then, these freeze-dried NPs were dispersed at a $\mathrm{Gd}^{3+}$ concentration of $500 \mathrm{mM}$ in ultrapure water and diluted tenfold with diethylene glycol (SDS Carlo Erba, France) at $80^{\circ} \mathrm{C}$ and then diluted twice with dry dimethyl sulfoxide (DMSO), leading to a final $25 \mathrm{mM}$ 
$\mathrm{Gd}^{3+}$. The carboxylic functions of the NPs were activated by $\mathrm{N}$-(3-Dimethylaminopropyl)-N0-ethylcarbodiimidehydrochloride (EDC) and pentafluorophenol (PFP) at EDC/PFP/Gd ${ }^{3+}$ molar ratio $10 / 20 / 1$ for $15 \mathrm{~min}$. PIB at $200 \mathrm{mg} \cdot \mathrm{ml}^{-1}$ in dry DMSO was added to the NPs suspension and the mixture was stirred for $6 \mathrm{~h}\left(\mathrm{PIB} / \mathrm{Gd}^{3+}\right.$ molar ratio $\left.1 / 1\right)$. Finally, the NPs were precipitated in acetone (Sodipro, France), centrifuged and the supernatant was removed; this step was repeated three-times to allow the removal of the upmost ungrafted PIB. After redispersion of the precipitate in water, acetone was evaporated overnight at $40^{\circ} \mathrm{C}$ in open vials. The unreacted reagents and the degraded NPs were efficiently eliminated by repeated concentration/dilution using tangential filtration cycles with a $5 \mathrm{kDa}$ molecular cut-off membrane. A full experimental characterization of the AGuIX@PEG@PIB samples is described in the Supplementary Information.

Functionalization of AGuIX NP with B10APB10AP was produced in $E$. coli and purified as described previously [31], from the plasmid ptetB10pA6H kindly provided by Prof Horn, Jena University, Germany. Freezedried AGuIX with 7\% of free DOTA (AGuIX@ DOTA $_{\text {free } 7 \%}$ ) were first labeled with one Cy5.5 dye molecule for $1000 \mathrm{Gd}^{3+}$ as previously described [23], and then were dispersed at $100 \mathrm{mM}$ in $\mathrm{Gd}^{3+}$ in ultrapure water. The carboxylic acid functions of the free DOTA ligand were activated using a 100-fold excess of EDC and N-hydroxysuccinimide. After 15 min of incubation, at room temperature under mild agitation, the activated AGuIX NPs were added in large excess (100fold excess) to the nanobody suspension at $41 \mu \mathrm{M}$ in phosphate-buffered saline buffer. Ungrafted AGuIX were also added to the suspension in order to reach a $\mathrm{Gd}^{3+}$ concentration of $10 \mathrm{mM}$ to stabilize grafted NPs. After $30 \mathrm{~min}$ of incubation, the ungrafted NPs are removed by tangential filtration on a $50 \mathrm{kDa}$ cut-off membrane. Then, the AGuIX@B10AP@Cy5.5 were freeze-dried and stored away from light. A full experimental characterization of the AGuIX@B10AP@Cy5.5 samples is described in the Supplementary Information.

EDC, PFP, PEG, N-hydroxysuccinimide (NHS) and DMSO were purchased from Aldrich Chemical (France).

\section{Amyloid fibril formation}

$\mathrm{A} \beta_{(1-42)}$ fibrils were prepared from $\mathrm{A} \beta_{(1-42)}$ peptide (Genecust, Luxembourg), incubated at $100 \mu \mathrm{M}$, and the V30M-TTR fibrils from recombinant protein produced in E. coli and were prepared at $32.3 \mu \mathrm{M}$, as previously described [23].

IAPP fibrils $(48 \mu \mathrm{M})$ were prepared using lyophilized IAPP peptide (Genecust) dissolved in 100\% 1,1,1,3,3,3-Hexafluoro-2-propanol (HFIP) and then incubated in Tris- $\mathrm{HCl} 20 \mathrm{mM} \mathrm{pH} 7.4$ at $37^{\circ} \mathrm{C}$ for 5 days, based on previous studies [34]. A full experimental detail of IAPP fibrils is described in the supplementary information.

The fibrillar morphology of the aggregates formed by the three peptides or proteins was confirmed using Transmission Electronic Microscopy analysis (JEOL1200EX/Tecnai 12).

\section{In vitro assays \& equilibrium constant determination}

Dissociation constants (i.e., affinities) were obtained by partition binding tests between fibrils and functionalized NPs as previously described [23]. Each type of fibrils, were incubated at 20 or $40 \mu \mathrm{M}$, with the following ranges of concentrations for AGuIX@ PEG@PIB, between 0.01 and $4 \mu \mathrm{M}$ in PIB, and for AGuIX@B10AP@Cy5.5 between 0.16 and 132 nM in B10. According to the fluorescence properties of PIB $\left(\lambda_{\mathrm{ex}}=350 \mathrm{~nm}, \lambda_{\mathrm{em}}=420 \mathrm{~nm}\right)$ or Cy5.5 $\left(\lambda_{\mathrm{ex}}=655 \mathrm{~nm}\right.$, $\lambda_{\text {em }}=685 \mathrm{~nm}$ ), fluorescence emission spectrum of each sample was recorded on a JASCO J-815 spectrophotometer. Michaelis binding model was used to calculate the equilibrium constant affinity $\left(K_{D}\right)$ following the equation:

$\mathrm{y}=\mathrm{Ym} /\left(1+\left(\mathrm{K}_{\mathrm{D}} / \mathrm{x}\right)\right)$

where $(\mathrm{Ym})$ is the fluorescence intensity maximum and $(y)$ is the fluorescence intensity measured for a given concentration in targeting probes within functionalized NPs (x).

\section{Animal models}

Brain sections prepared from 18 months APPswe/ PSEN1dE9 AD mouse models (kindly provided by $\mathrm{M}$ Dhenain, Fontenay aux Roses, France) were used to target A $\beta$-peptide fibrils. These models were previously described in the literature [35,36], and a short description is provided within Supplementary Information. Brain sections from C57/B16 mice were used as control.

Pancreas sections were prepared from male mice homozygous for RIPHAT transgene-expressing human-IAPP as TDM2 models [37]. Pancreas from S/ $\mathrm{FVB} / \mathrm{NJ}$ mice were used as control tissue without amyloid burden.

Stomach tissue sections were extracted from a transgenic mice expressing human V30M-TTR and lacking endogenous mouse TTR [38]. Similar tissue from knockdown mice for transthyretin gene was used as control [39].

All the procedures involving animals were carried out in accordance with the European Community Council Directives. The different procedures to deeply anesthetize the animals, to extract and fix the organs/ 
tissues and to prepare the sections are described in details within Supplementary Information. Tissue sections were mounted on slides for the immunostaining experiments.

\section{Immunohistochemistry}

Frozen sections were processed for immunohistochemistry staining with AGuIX@PEG@PIB and AGuIX@ B10AP@Cy5.5 as previously described with other functionalized AGuIX [23].AGuIX@PEG@PIB were used at $(\mathrm{PIB})=5 \mu \mathrm{M}$ and AGuIX@B10AP@cy5.5 at (B10) $=81 \mathrm{nM}$. To each suspension of grafted NPs, $3 \mathrm{mM}$ of ungrafted NPs were added to insure the stability of the former. In the case of PIB, fluorescence was used to monitor the binding of AGuIX@PEG@PIB $\left(\lambda_{\text {ex }}=\right.$ $\left.350 \mathrm{~nm}-\lambda_{\mathrm{em}}=405 \mathrm{~nm}\right)$. TRITC-conjugated phalloidin (Sigma, France, \#P1951) was used to detect the actin network $\left(\lambda_{\mathrm{ex}}=545 \mathrm{~nm}-\lambda_{\mathrm{em}}=605 \mathrm{~nm}\right)$. Regarding AGuIX@PEG@B10AP@Cy5.5, the binding to NPs to amyloid plaques was detected through either the fluorescence of Cy5.5 $\left(\lambda_{\mathrm{ex}}=650 \mathrm{~nm}-\lambda_{\mathrm{em}}=670 \mathrm{~nm}\right)$ or via the enzymatic activity of AP fused to B10AP using red permanent as substrate $\left(\lambda_{\text {ex }}=595 \mathrm{~nm}-\lambda_{\text {em }}=613 \mathrm{~nm}\right)$. In all tissue sections, amyloid burden was stained with $\mathrm{ThS}\left(\lambda_{\text {ex }}=450 \mathrm{~nm}-\lambda_{\text {em }}=488 \mathrm{~nm}\right)$ (Sigma, \#T1892) according to standard practice. Fluorescent images were recorded using either Zeiss confocal LSM800 microscope for pancreas sections or Zeiss microscope Axiovert $200 \mathrm{M}$ for brain and stomach tissues. Images were analyzed using Carl Zeiss AxioVision software and Image J $1.50 \mathrm{f}$ software.

\section{Results}

\section{Characterization of the grafted NPs}

The synthesis of functionalized AGuIX NPs was achieved through a bottom-up process described previously $[18,19]$, with around ten DOTAGA per NP. Two different strategies were used to graft PIB and B10AP, mainly due to their sizes (Figure $1 \mathrm{~A}$ ). The addition of small PEG chains at the surface of AGuIX was necessary to guarantee the accessibility of PIB (i.e., to limit the steric hindrance due to the proximity of the NP) for the interaction with amyloid aggregates. The hydrodynamic diameter $\left(\mathrm{D}_{\mathrm{H}}\right)$ of AGuIX increased from $2.9 \pm 0.7 \mathrm{~nm}$ to $4.3 \pm 1.0 \mathrm{~nm}$ after addition of PEG chains and to $6.5 \pm 1.5 \mathrm{~nm}$ after PIB grafting (Figure 1B, Table 1). In addition, the relaxometric characterization $r_{1}$ slightly increased from naked AGuIX to AGuIX@PEG@PIB (Supplementary Table 1), from $10 \mathrm{mM}^{-1} \mathrm{~s}^{-1}$ to $12.9 \mathrm{mM}^{-1} \mathrm{~s}^{-1}$. The number of molecules of PIB grafted on NPs was estimated by absorption spectroscopy (Figure 1C). A standard curve, in other words, absorbance at $338 \mathrm{~nm}\left(\mathrm{~A}_{338}\right)$ as a function of (PIB), was first established with known solutions of
PEG@PIB. The optical properties of PIB are sensitive to the solvent polarity, thus the standard curve was established taking into account $[40,41]$. Then, the PIB concentrations were estimated for two dilutions of the AGuIX@PEG@PIB stock solution (known $\left[\mathrm{Gd}^{3+}\right]$ ) to get the ratio between PIB and $\mathrm{Gd}^{3+}$. Then, the PIB concentrations were estimated for two dilutions of the AGuIX@PEG@PIB stock solution to get the ratio between PIB and $\mathrm{Gd}^{3+}$ : it was evaluated to one PIB for $50 \mathrm{Gd}^{3+}$.

The strategy to graft B10AP was to use NPs displaying about $7 \%$ of free DOTA. B10AP was then directly bound to free carboxylic acid functions of these DOTA (Figure 1A). To allow their detection by fluorescence microscopy, Cy 5.5 was grafted on the NPs before grafting B10AP. The hydrodynamic diameter for AGuIX@

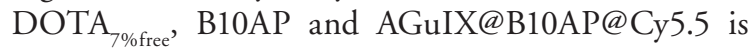
$4.2 \pm 0.7 \mathrm{~nm}, 9.2 \pm 2.9 \mathrm{~nm}$ and $13.9 \pm 2.6 \mathrm{~nm}$, respectively (Figure 1D). The concentration of B10AP within the NPs suspension was estimated by SDS-PAGE. A standard curve was built with a series of known B10AP suspensions (see 'Materials \& Methods' within the Supplementary Information). Then, a series of three dilutions of AGuIX@B10AP@Cy5.5 stock solution was used to estimate the concentration of B10AP and thus estimate the ratio between (B10AP) and $\left(\mathrm{Gd}^{3+}\right)$. This later was evaluated at $1 \mathrm{~B} 10 \mathrm{AP}$ for $54 \mathrm{Gd}^{3+}$, in other words, 1 B10AP for 5.4 NPs (Figure 1E \& Table 1).

\section{Affinities of functionalized NPs for amyloid fibrils}

The formation of $A \beta_{(1-42)}$, IAPP and V30M-TTR amyloid fibrils in vitro was assessed by Transmission Electronic Microscopy (Figure 2). NP-fibril interactions were monitored by partition assays. Amyloid fibrils and NPs were incubated for $1.5 \mathrm{~h}$; then, the NPs bound to amyloid fibrils were separated from the free ones by centrifugation, and the supernatants and the pellets were collected separately. At last, fluorescence spectra was recorded for the supernatants and the resuspended pellets. The fact that the sum of these two spectra was close to that of the suspension before centrifugation indicated that no NPs were lost during the experiments (Figures 3A \& 4A). The fluorescence intensity of the pellets increased with the NP concentration at constant concentration of amyloid fibrils, showing that we were able to monitor the binding of the NPs (Figures 3B-D \& 4B-D).

In the case of AGuIX@PEG@PIB (Figure 3B-D), the fluorescence changes at $420 \mathrm{~nm}$ allowed to estimate $K_{D}$ values of these NPs for the three types of amyloid fibrils. We were not able to use NP concentrations high enough to reach a plateau for the binding (Figure 3B-D). However, the use of a simple Michaelis 

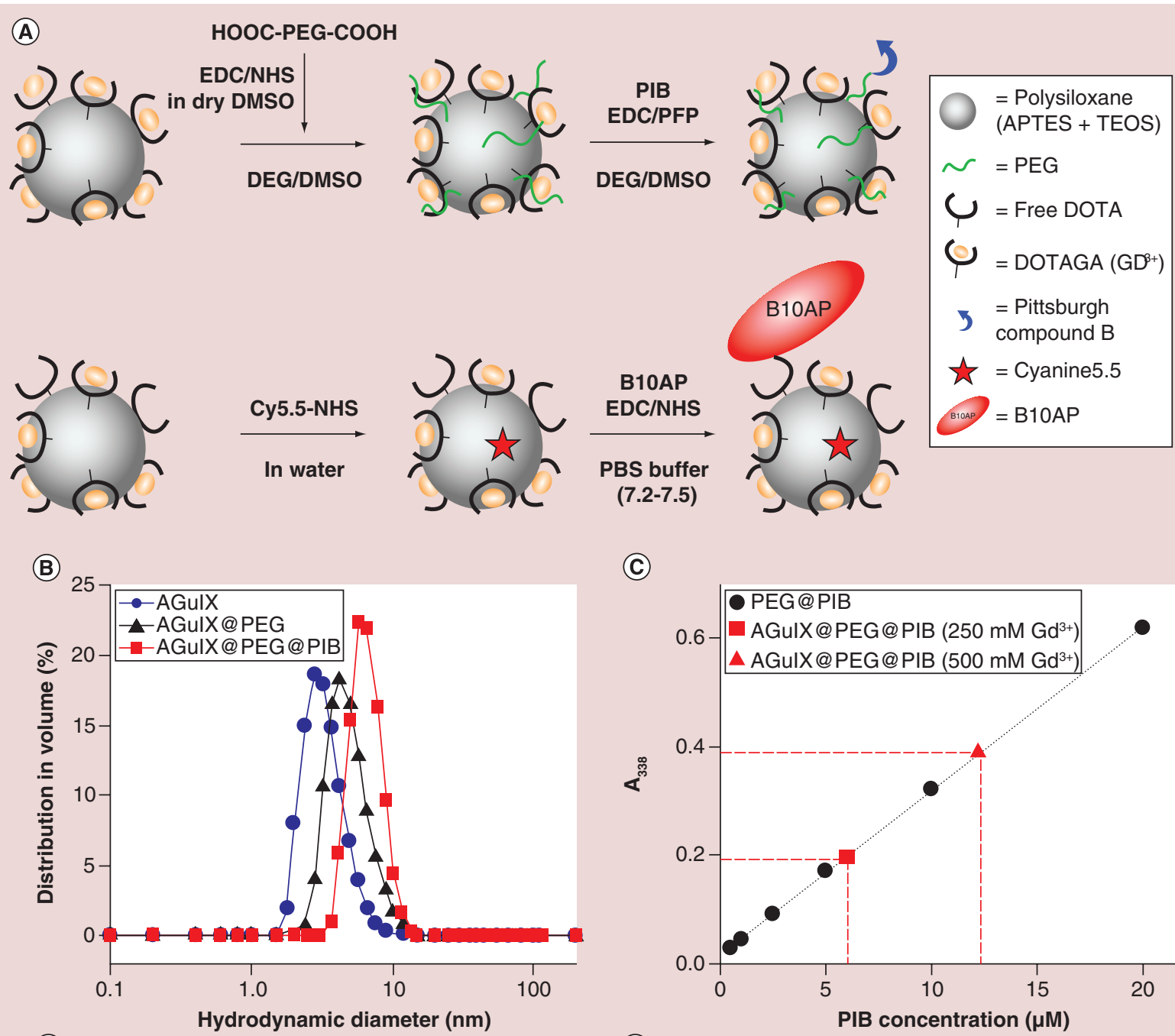

(C)

(D)
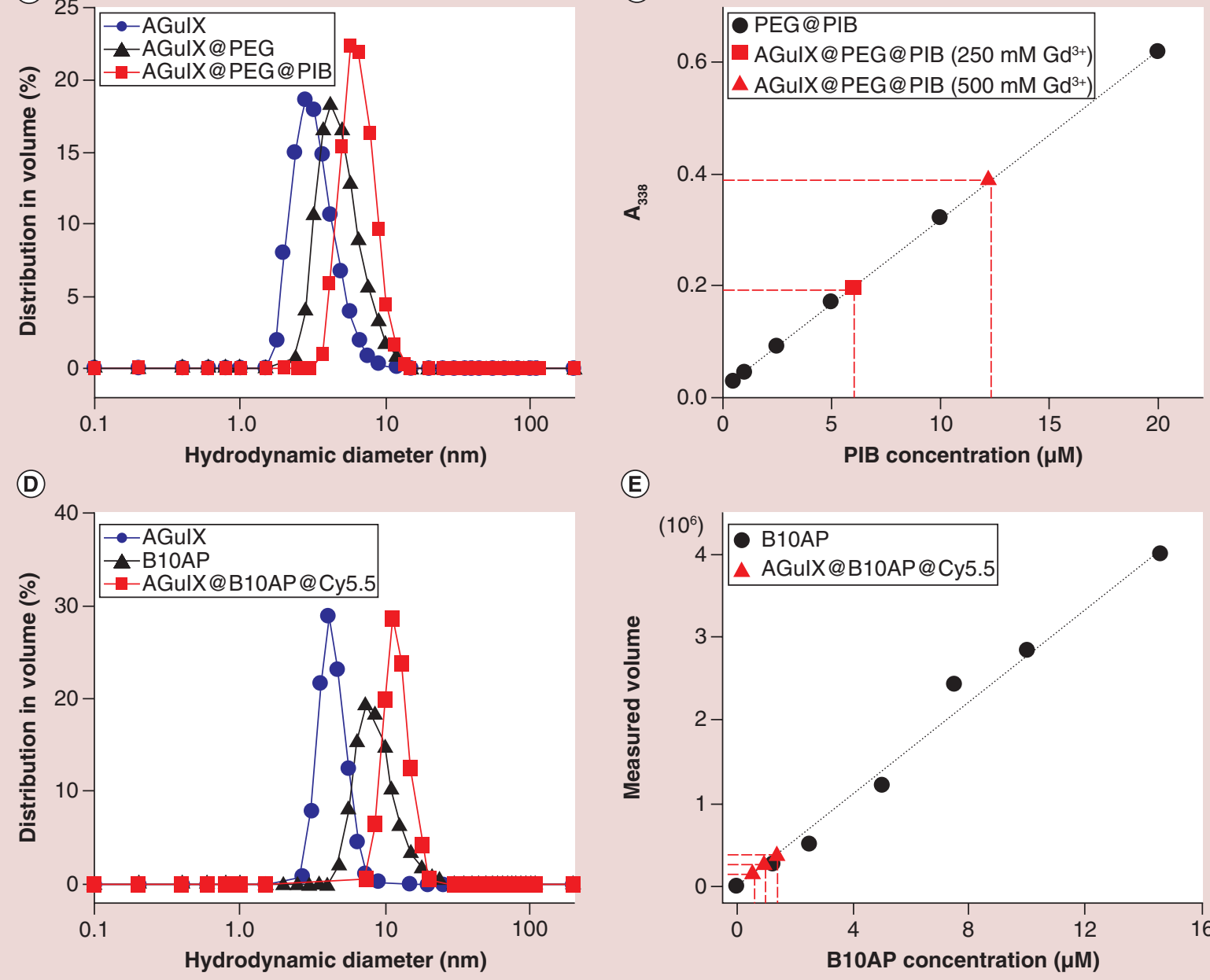

(E)

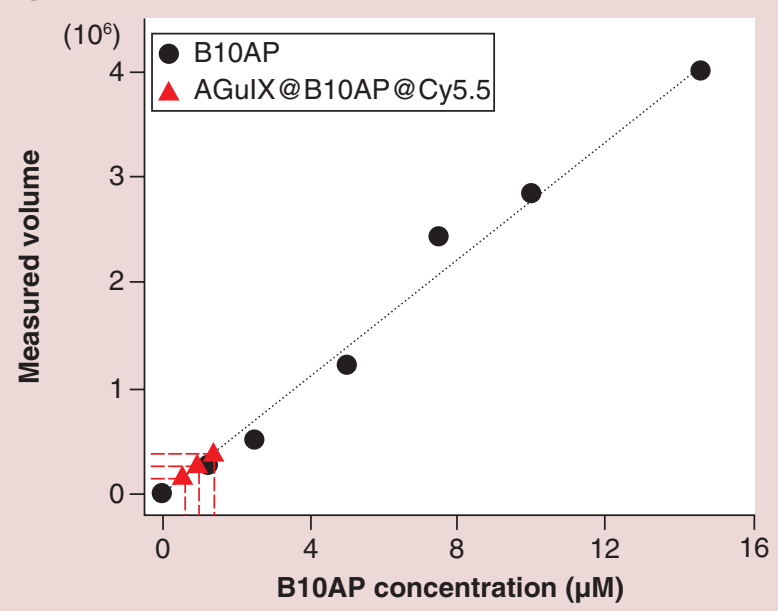

Figure 1. Synthesis and characterization of AGuIX@PEG@PIB and AGuIX@B10AP@Cy5.5 a. Reaction schemes of AGulX nanoparticles PEGylation followed by PIB grafting (upper scheme) and AGuIX cyanination followed by the grafting of B10AP (lower scheme).

(B) Dynamic light scattering measurements of AGuIX (blue circle), AGuIX@PEG (black triangle) \& AGuIX@PEG@PIB nanoparticles (red square). (C) Absorption quantification of PIB grafted on AGuIX@PEG (red markers) using standard curve of PEG@PIB (black circle) at

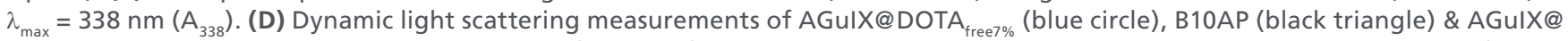
B10AP@Cy5.5 (red square). (E) SDS-PAGE quantification of B10AP in AGuIX@B10AP@Cy5.5 suspension using standard curve of B10AP. DMSO: Dimethyl sulfoxide; EDC: N-(3-Dimethylaminopropyl)-N0-ethylcarbodiimidehydrochloride; NHS: N-hydroxysuccinimide; PBS: Phosphate-buffered saline; PFP: Pentafluorophenol; PIB: Pittsburgh compound B. 


\begin{tabular}{|c|c|c|c|c|c|}
\hline & AGuIX & AGuIX@PEG & $\begin{array}{l}\text { AGulX@PEG@ } \\
\text { PIB }\end{array}$ & $\begin{array}{l}\text { AGuIX } \\
\text { (DOTA }_{\text {free7\% }} \text { ) }\end{array}$ & $\begin{array}{l}\text { AGulX@B10AP@ } \\
\text { Cy5.5 }\end{array}$ \\
\hline $\begin{array}{l}\text { Hydrodynamic } \\
\text { diameter }\left(D_{H}\right)\end{array}$ & $2.9 \pm 0.7$ & $4.3 \pm 1.0$ & $6.5 \pm 1.5$ & $4.2 \pm 0.7$ & $13.9 \pm 2.6$ \\
\hline $\begin{array}{l}\text { Quantification } \\
\text { of grafting }\end{array}$ & - & - & 1 PIB:5 NPs & - & 1 B10AP:5.4 NPs* \\
\hline
\end{tabular}

model, which assumed that the binding was noncooperative, allowed a fair estimation of the $\mathrm{K}_{\mathrm{D}}: 15 \pm 3 \mu \mathrm{M}$ for $\mathrm{A} \beta_{(1-42)}$ fibrils, $21 \pm 8 \mu \mathrm{M}$ for IAPP fibrils and $57 \pm$ $27 \mu \mathrm{M}$ for V30M-TTR fibrils. In the case of AGuIX@ B10AP@Cy5.5 (Figure 4B-D), the $\mathrm{K}_{\mathrm{D}}$ values are: $46 \pm 7$ $\mathrm{nM}$ for $\mathrm{A} \beta_{(1-42)}, 21 \pm 4 \mathrm{nM}$ for IAPP and $264 \pm 51 \mathrm{nM}$ for V30M-TTR fibrils.

For all NPs tested, we checked that the binding was really due to B10AP and PIB by carrying out partition experiments with NPs but in the absence of fibrils. The results of these experiments showed that no significant amount of NPs was pelleted due to aggregation (Supplementary Figure 2A). On the other hand, less than $5 \%$ of total fluorescence was measured in pellet for the partition experiments carried out with AGuIX@ Cy5.5 and amyloid fibrils (Supplementary Figure 2B). These results clearly indicated that there was no interaction between amyloid fibrils and NPs in the absence of the amyloid probes. All together, these results indicated that both NPs specifically bound with the three types of amyloid fibrils tested, via the amyloid probe, and that B10AP NPs had a better affinity for amyloid fibrils compared with PIB NPs.

Tissue amyloid burden targeting

Then the capacity of each NP to detect amyloid bur- den was tested on ex vivo tissue sections from mice model of each pathology: the brain of $\mathrm{AD}$ mice (Figure 5A-E, Figure 6A-E), the pancreas of T2DM mice (Figure $5 \mathrm{~F}-\mathrm{G}$, Figure $6 \mathrm{~F}-\mathrm{G}$ ) and the stomach of FAP mice (Figure $5 \mathrm{H}-\mathrm{I}$, Figure $6 \mathrm{H}-\mathrm{I}$ ). In $\mathrm{AD}$ mice brain, amyloid burdens are expected mainly within the hippocampus and the cortex [42]. Spots exhibiting strong PIB fluorescence were detected within the hippocampus on an AD brain slice imaged with AGuIX@PEG@ PIB (Figure 5C; blue color). They co-localized with ThS staining (Figure 5D; green color). A staining with phalloidin was also carried out to visualize the different structures by labeling actin networks (Figure 5E). The merged image highlighted the co-localization of AGuIX@PEG@PIB and ThS staining, demonstrating that AGuIX@PEG@PIB selectively recognized the amyloid burden (Figure 5A). On pancreas sections from T2DM mice, PIB and ThS signal also co-localized and were detected within selective and discrete areas at the level of Langherans islets, as expected for this type of model tissue [13] (Figure 5F). A similar colocalization was also observed with stomach sections of FAP mice (Figure 5H) where V30M-TTR deposits have been described [43]. No fluorescence, and therefore no staining, was observed when similar imaging studies were carried out on control tissue sections,
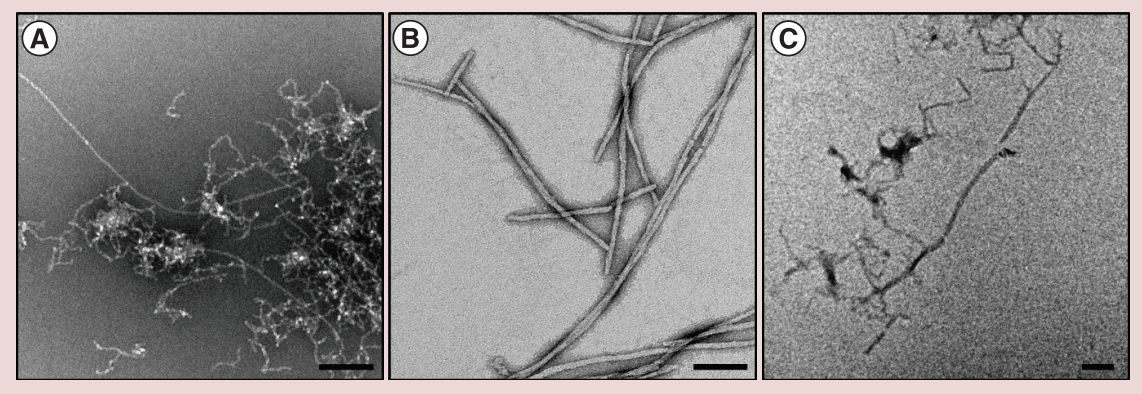

Figure 2. Transmission electron microscopy of amyloid fibrils. TEM pictures for $A \beta(1-42)(A), I A P P(B)$ and V30MTTR (C) fibrils. Fibrils with mean apparent diameter of respectively 5, 10 and $12 \mathrm{~nm}$ for A $\beta_{(1-42)}$, IAPP and V30M-TTR are observed. Scale bar $100 \mathrm{~nm}$. IAPP: Islet amyloid polypeptide. 


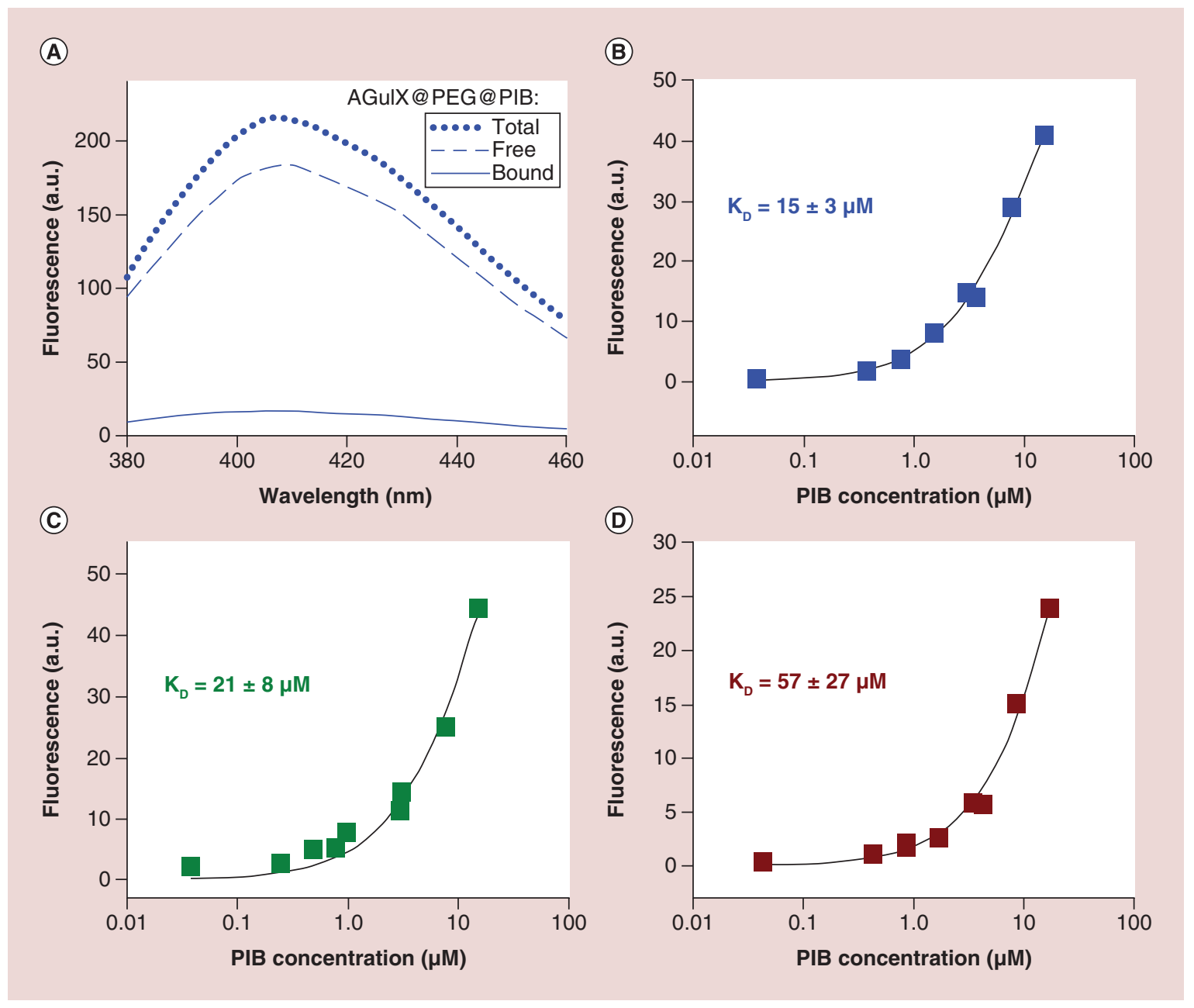

Figure 3. Interaction in vitro between amyloid fibrils and AGuIX@PEG@PIB by partition experiments. (A) A total of 800 nM in PIB of AGuIX@PEG@PIB was added to $20 \mu \mathrm{M}$ of $A \beta_{(1-42)}$ fibrils and the fluorescence of the suspension was measured (dotted line); then after 60 min of incubation, the suspension was centrifuged and the fluorescence of NPs bound to the amyloid fibrils (i.e., present in the pellet, solid line) and that of unbound NPs (i.e., present in the supernatant, dotted line) were measured (B, C \& D). Determination of the $K_{D}$ using Michaelis model (1:1). The fluorescence intensity of NPs bound at approximately $420 \mathrm{~nm}$ is plotted as function of the total concentration of PIB functionalized on NPs, with $A_{(1-42)}(B)$, IAPP (C) or V30M-TTR (D). The solid line represents the adjustment of the data to the Michaelis model $(1: 1)$ increasing concentration of PIB and fitted with.

IAPP: Islet amyloid polypeptide; NP: Nanoparticle; PIB: Pittsburgh compound B.

prepared from control mice who do not develop the diseases (Figure 5B, G \& I). All together, these results clearly demonstrate the AGuIX@PEG@PIB selectively labeled amyloid plaques on tissues from the mice model of the three pathologies investigated.

Similar experiments indicated that AGuIX@ B10AP@Cy5.5 also specifically stained amyloid deposits on the three types of tissues (Figure 6A, F, H). Moreover, the binding of AGuIX@B10AP@Cy5.5 can be detected through both the activity of AP (Figure 6C; red color) and the fluorescence of Cy5.5 (Figure 6D; yellow color). In brain sections of AD mice, these signals co-localized with ThS labeling (Figure 6E; green color) within the hippocampus. On the merged picture
(Figure 6A), the three signals co-localized and resulted in intense yellow spots. Such co-localization of the three type of staining was also observed in pancreas tissue, and in particular in the Langherans islets of T2DM mice (Figure 6F). Note that a labeling of the cellular nucleus with Hoechst dye was also carried out on these later samples in order to easily identify Langherans islet (Figure 6F \& G; blue color). Finally, with stomach tissue sections of FAP mice, a signal typically resulting from the co-localization of AGuIX@B10AP@Cy5.5 with ThS was also detected and assigned to amyloid deposits (Figure 6H). Finally, the specificity of the binding of AGuIX@B10AP@Cy5.5 to amyloid was confirmed by the absence of a fluorescence signal for similar experi- 


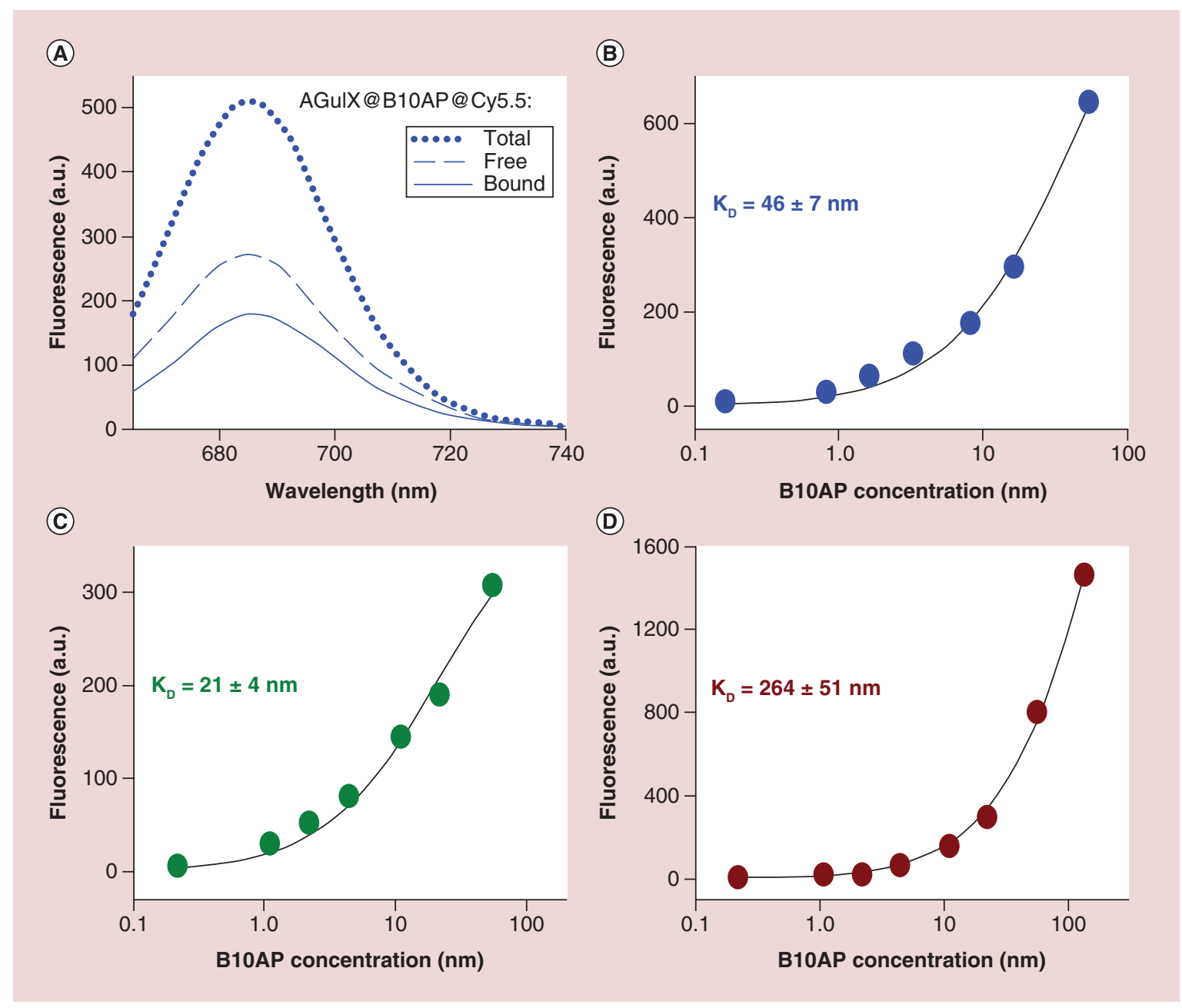

Figure 4. Interaction in vitro between amyloid fibrils and AGuIX@B10AP@cy5.5 by partition experiments. (A) A total of $11 \mathrm{nM}$ in B10AP of AGuIX@B10AP@Cy5.5 was added to $40 \mu \mathrm{M}$ of A $\beta(1-42)$ fibrils and the fluorescence of the suspension was measured (dotted line); then after $60 \mathrm{~min}$ of incubation, the suspension was centrifuged and the fluorescence of NPs bound to the amyloid fibrils (i.e., present in the pellet, solid line) and that of unbound NPs (i.e., present in the supernatant, dotted line) were measured (B, C \& D). Determination of the $K_{D}$ using Michaelis model (1:1). The fluorescence intensity of NPs bound at approximately $685 \mathrm{~nm}$ (Cy5.5 fluorescence) is plotted as function of the total concentration of B10AP functionalized on NPs, with A $\beta_{(1-42)}$ (B), IAPP (C) or V30M-TTR (D). The solid line represents the adjustment of the data to the Michaelis model (1:1) increasing concentration of B10AP and fitted with.

IAPP: Islet amyloid polypeptide; NP: Nanoparticle.

ments carried out on control tissues which did not contain amyloid deposition (Figure 6B, G \& I).

\section{Discussion}

The aim of this work was to develop a specific, versatile and noninvasive tool detectable by medical imaging able to specifically target amyloid deposits for a more sensitive and early diagnostic of amyloidosis.

One of the important features of functionalization of NPs is the control of the synthesis in terms of chemical reaction, quality of grafting and size of the final product. The grafting of PIB to AGuIX NPs increases their hydrodynamic diameter (Table 1). However, their size remains homogenous (Figure $1 \mathrm{~A}$ ) and their relaxometric characterization shows good relaxivity at 12.9 $\mathrm{mM}^{-1} \cdot \mathrm{s}^{-1}$ allowing MRI detection. Therefore, the $\mathrm{Gd}^{3+}$ relaxation properties of the NPs are not altered by the grafting.

The affinity of AGuIX@PEG@PIB for amyloid fibrils is approximately $10 \mu \mathrm{M}$, close to the value that was previously reported for PIB alone [23]. The addition of the PEG linker between PIB and the NP allows PIB to be sufficiently accessible to interact with its target. One can note here, that the dissociation constants, that have been measured for PIB in this work, are several orders of magnitude higher than those reported 

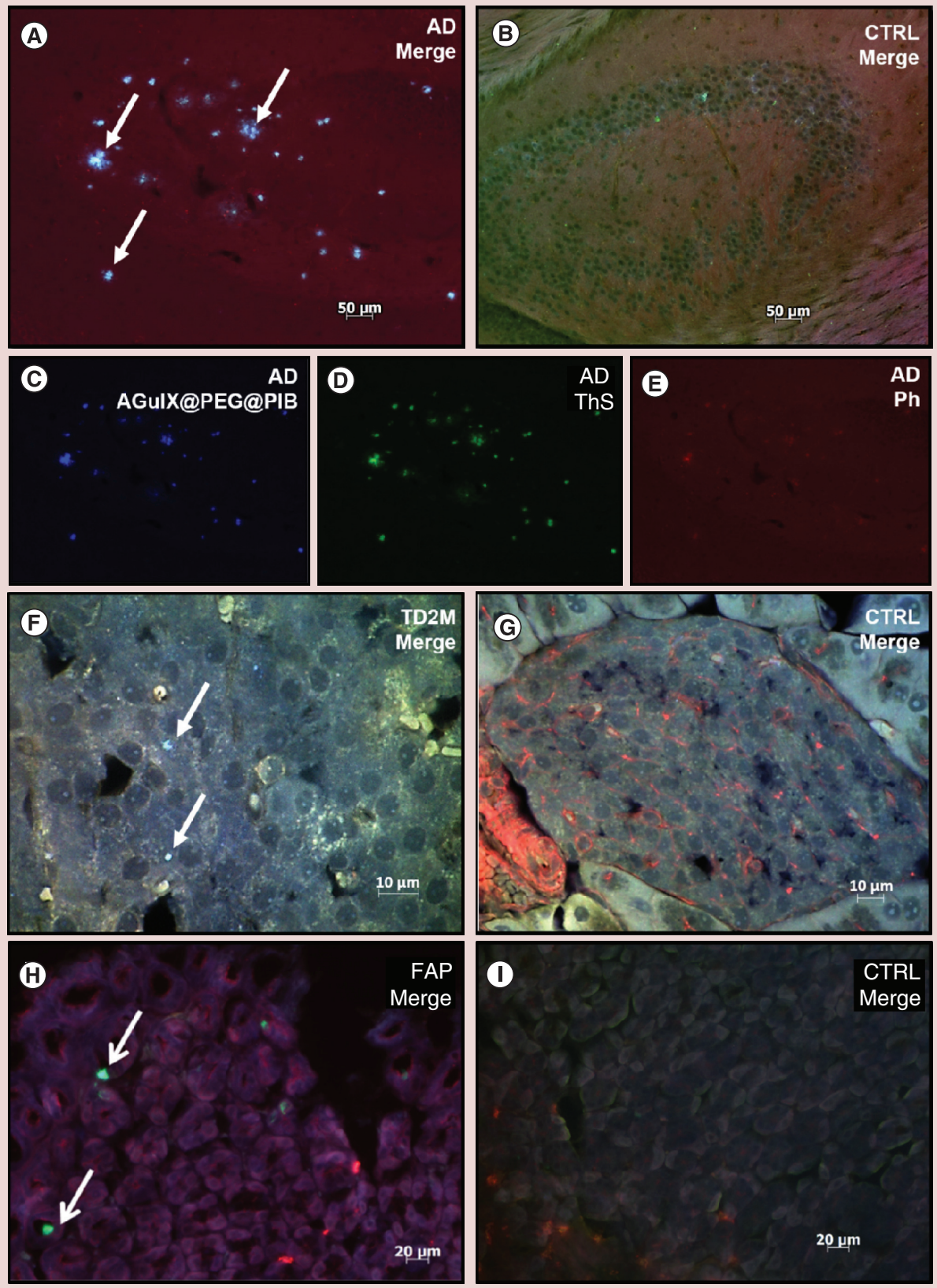

Figure 5. Amyloid deposit targeting with AGuIX@PEG@PIB in pathological tissue. Specific targeting of amyloid plaques with AGuIX@PEG@PIB nanoparticles on brain sections of APPswe/PSE1d9 mice (A, C-E), on pancreas sections of S/FVB/N-Tg/(ins2-hIAPP) mice (F) or stomach sections of transgenic V30M-TTR mice (H) and on respective negative control tissues: C57/BI6 mice (B), S/FVB/NJ mice (G) and KO-TTR mice (I). Nanoparticles targeting were detected via the PIB fluorescent signal (C, blue color). Amyloid plaques were visualized using Thioflavin-S staining ( $D$, green color). The co-localization between nanoparticles and amyloid plaques were obtained by merging PIB and ThS fluorescent staining (A, F \& H, white arrows). TRITC-conjugate of phalloidin was used to label cellular actin filament ( $E$, red color).

AD: Alzheimer's disease; CTRL: Control; FAP: Familial amyloidotic polyneuropathy; IAPP: Islet amyloid polypeptide; NP: Nanoparticle; PIB: Pittsburgh compound B; TD2M: Type 2 diabetes mellitus; ThS: Thioflavin; TRITC: Tetramethylrhodamine.

in the literature [11,25-26]. The reasons for this apparent discrepancy are unknown, but the conditions and the methods used in our experiments are drastically different. As PIB has kept both its optical properties 


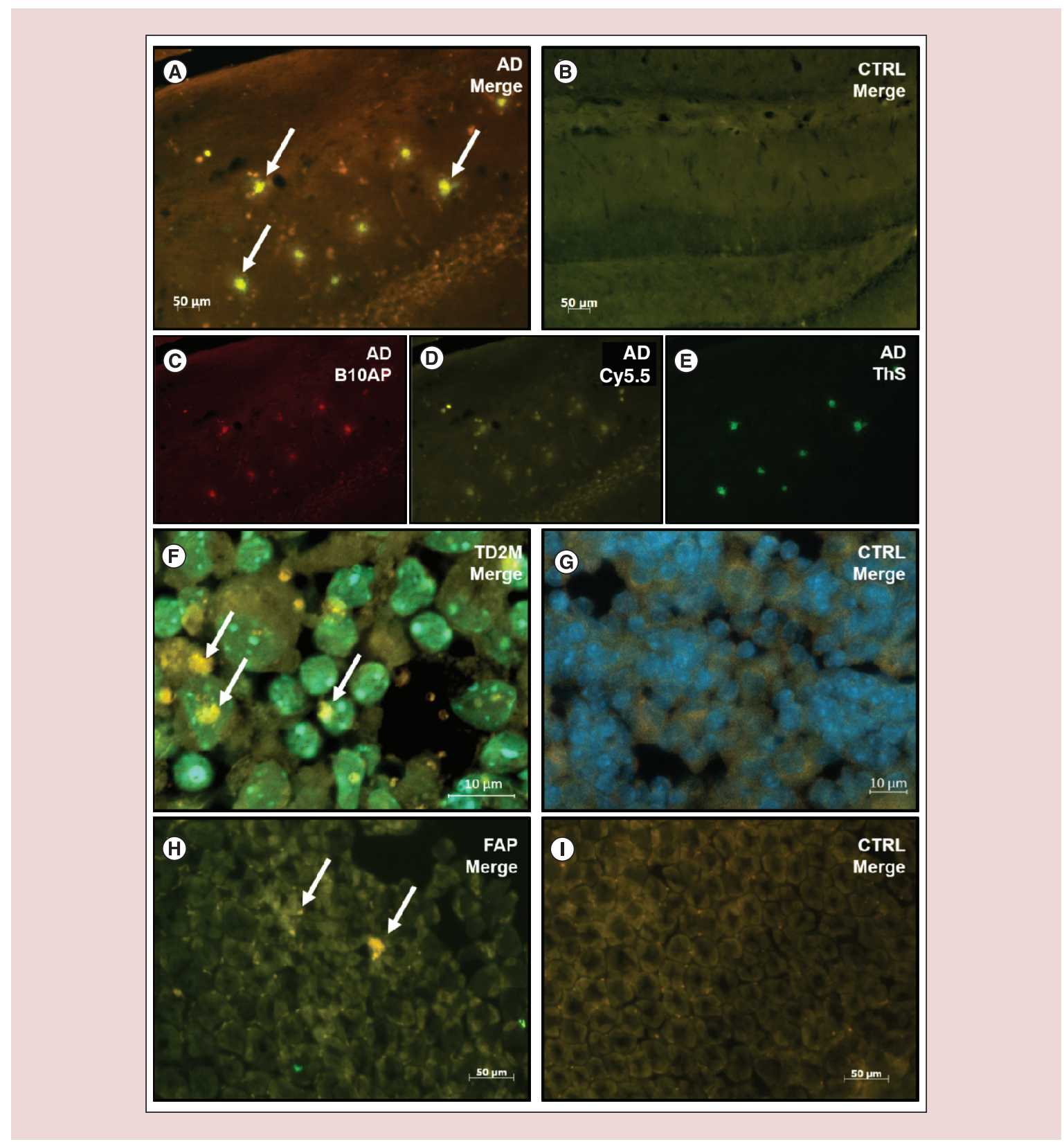

Figure 6. Amyloid deposit targeting with AGuIX@PEG@Cy5.5 in pathological tissue. Specific targeting of amyloid plaques with AGuIX@B10AP@Cy5.5 nanoparticles on brain slices of APPswe/PSE1d9 mice (A, C-E), on pancreas sections of S/FVB/N-Tg/(ins2-hIAPP) mice (F) or stomach section of transgenic V30M-TTR mice (H) and on respective sections of negative control tissues: C57/BI6 mice (B), S/FVB/NJ mice (G) and KO-TTR mice (I). Nanoparticles targeting were detected due to both red permanent reaction with alkaline phosphatase (C, red color) and Cy5.5 fluorescent signal (D, yellow color). Amyloid plaques were visualized using Thioflavin-S dye staining ( $E$, green color). The co-localization between nanoparticles and amyloid plaques were obtained by merging red permanent, Cy5.5 and Thio-S fluorescent staining (A, F, H, white arrows). Cellular nucleus of the tissue were stained with Hoechst dye (F, G, blue color).

AD: Alzheimer's disease; CTRL: Control; FAP: Familial amyloidotic polyneuropathy; TD2M: Type 2 diabetes mellitus.

and its ability to recognize amyloid aggregates, the multimodality of the NPs has been confirmed.

A further milestone for the development of new multimodal NPs has been reached with the successful grafting of B10AP. This was a challenge because of constraints implied by the use of a multidomain protein. The affinity of AGuIX@B10AP@Cy5.5 for both $\mathrm{A} \beta$ and IAPP fibrils is approximately $10 \mathrm{nM}$ (Figure $4 \mathrm{~B}-\mathrm{C}$ ), and is comparable both to that measured with B10AP alone (8.3 nM for $A \beta_{(1-42)}$, not shown) 
and to that reported in the literature [32]. These results clearly demonstrate that B10 remains functional upon grafting to the NPs. Therefore, AGuIX NP may also constitute an interesting platform for high molecular weight molecules.

The ability of the functionalized NPs to stain amyloid fibrils on ex vivo tissues, was investigated by immunohistochemistry experiments performed on pathological tissues from mice model of each disorder.AGuIX@PEG@PIB or AGuIX@B10AP@Cy5.5 fluorescence is observed in all tested pathological tissues on distinct amyloid spots (Figures 5 \& 6). In such experiments, the co-localization of Cy5.5 fluorescence and AP activity signal in the same restricted zones was a further proof of the integrity of AGuIX@B10AP@ Cy5.5. In addition, the fact that both NPs co-localized with Thioflavin-S staining, confirms their specific targeting of amyloid burden.

The determination of the equilibrium dissociation constant of these NPs is important for estimating their potential as diagnosis tools. The affinity for amyloid fibrils of AGuIX@PEG@PIB is in the $10 \mu \mathrm{M}$ range and that of AGuIX@B10AP@Cy5.5 in the 10 nanomolar range. Such values are compatible with the use of such NP for in in vivo studies (Figures $3 \& 4$ ). The dissociation constant of AGuIX@B10AP@Cy5.5 for V30M-TTR fibrils is significantly larger than for the other two types of fibrils. The reason for this lower affinity is unknown. However, it has been reported that B10 does not recognize all amyloid fibrils with the same efficiency [32]; this compromises its use for diagnostic. This work, however, clearly demonstrates that large multidomain proteins can be efficiently linked to AGuIX; this proof of concept opens the possibility to functionalize these NPs with nanobodies recognizing selective epitopes of the $\mathrm{AD}$ at the surface of extracellular amyloid plaque or intracellular targets [44]. Moreover, small peptides with high affinity $\left(K_{D}\right.$ in 10 nanomolar range $\left.[45,46]\right)$ for a generic epitope at the surface of $A \beta$ amyloid fibrils have been recently described and could also constitute valuable probes to be grafted on AGuIX. Nevertheless, these peptides present important drawbacks since they induce cell toxicity [45] or stabilize protofibrils [46]. Some other peptides specifically targeting $A \beta$-peptide fibrils with no toxic effects have been described but their affinities are too low for in vivo imaging (100 micromolar range) [23].

\section{Conclusion}

In this work, we have shown that AGuIX can be successfully functionalized with a small molecule and a large protein to specifically target amyloid fibrils. Thus, the NPs tested in this work provide complementary tools that could be useful for in vivo diagnostic: strong affinity of AGuIX@B10AP@Cy5.5 will be helpful for early amyloid deposits detection, when the smaller size of AGuIX@PEG@PIB should diffuse more quickly, particularly interesting notably for systemic amyloidosis (V30M-TTR). In the case of AD diagnostic, we must take into account that such NPs have to reach their target inside the brain, implicating the crossing of the hermetic blood-brain barrier. However, the flexibility of AGuIX NPs may allow chemical modifications for diffusion through this barrier or addition of molecules to improve active transport through specific receptor of the blood-brain barrier.

In any case, such functionalized NPs are very promising to target several types of amyloid deposits in vivo, because of their very low levels of toxicity; their rapid clearance [47]; and the divert and complementarity of imaging techniques with great sensitivity and spatial resolution that can be used.

\section{Acknowledgements}

We thank F Daphna, M Christine and S Guy from the Electron Microscopy platform of the Integrated Structural Biology of Grenoble (ISBG, UMI3265). Others TEM images were obtained at the electronic microscopy platform, Neurosciences Institute of Grenoble thanks to K Pernet Gallay, 38700 Grenoble. The authors thank Horn from Jena University (Germany) for the kind gift of the plasmid ptetB10pA6H. The authors thank $R$ Cohen for his helpful expertise in affinity experiments.

\section{Financial \& competing interests disclosure}

This work was supported by EuroNanoMed ENMII JTC2012 (project 2011-ERA-002-01- Dia-Amyl) and the French National Research Agency (ANR): ANR-12-RPIB Multimage. J Pansieri is granted by FRM DBS2013112844. M Plissonneau and N Stransky-Heilkron were granted by ENMII JTC2012 (project 2011-ERA-002-01). M Dumoulin is an FRS-FNRS research associate. This work was also supported by the Belgian program of Interuniversity Attraction Poles administered by the Federal Office for Scientific Technical and Cultural Affairs to MD (PAI 7/44). F Lux and O Tillement have one patent to disclose: WO2011135101. This patent protects the synthesis of the $A G u l X{ }^{\circledR}$ nanoparticles for imaging, used in this publication. This work used the platforms of the Grenoble Instruct centre (ISBG; UMS 3518 CNRS-CEA-UJF-EMBL) with support from FRISBI (ANR-10-INSB-05-02) and GRAL (ANR-10LABX-49-01) within the Grenoble Partnership for Structural Biology (PSB). The authors have no other relevant affiliations or financial involvement with any organization or entity with a financial interest in or financial conflict with the subject matter or materials discussed in the manuscript apart from those disclosed.

No writing assistance was utilized in the production of this manuscript. 
Ethical conduct of research

The authors state that they have obtained appropriate institutional review board approval or have followed the principles outlined in the Declaration of Helsinki for all human or animal experimental investigations. In addition, for investigations involving human subjects, informed consent has been obtained from the participants involved.

Summary points

- To achieve the diagnostic of amyloidosis, multimodal imaging nanoparticles were functionalized with either the Pittsburgh compound B or a nanobody.

- Efficient active targeting was tested by spectroscopic and biophysics techniques on A $\beta_{(1-42)}$, IAPP and Val30MetTTR fibrils.

- Both nanoparticles efficiently interacted with the three types of amyloid fibrils, with $K_{D}$ values in 10 micromolar range for AGuIX@PIB and 10 nmolar range for AGuIX@Cya5.5@B10AP.

- Both functionalized nanoparticles are able to interact specifically with amyloid deposits localized in pathological tissue.

- These functionalized nanoparticles represent promising flexible devices for multimodal imaging in early diagnostic of amyloid plaques, in other words, Alzheimer's disease, Type 2 diabetes mellitus and the familial amyloidotic polyneuropathy.

\section{References}

Papers of special note have been highlighted as: $\bullet$ of interest; •• of considerable interest

1 Knowles TP, Vendruscolo M, Dobson CM. The amyloid state and its association with protein misfolding diseases. Nat. Rev. Mol. Cell Biol. 15(6), 384-396 (2014).

- Shows how much progress has been made toward knowledge of the amyloid phenomenon providing basis for therapeutic advances.

2 Tycko R. Solid state NMR studies of amyloid fibril structure. Annu. Rev. Phys. Chem. 62, 279-299 (2011).

3 Sipe JD, Benson MD, Buxbaum JN et al. Amyloid fibril proteins and amyloidosis: chemical identification and clinical classification International Society of Amyloidosis 2016 Nomenclature Guidelines. Amyloid 23, 209-213 (2016).

4 World Alzheimer Report 2014: Dementia and Risk Reduction | Alzheimer's Disease International. www.alz. co.uk/research/world-report-2014

5 IDF diabetes atlas - Home. www.diabetesatlas.org/

6 Perrin RJ, Fagan AM, Holtzman DM. Multimodal techniques for diagnosis and prognosis of Alzheimer's disease. Nature 461, 916-922 (2009).

7 Gilbertson JA, Botcher NA, Rowczenio D et al. Diagnostic value of fat aspirates for amyloidosis in 950 patients. Orphanet J. Rare Dis. 10(Suppl. 1), P50 (2015).

8 Liu PP, Smyth D. Wild type transthyretin amyloid cardiomyopathy: a missed etiology of HFpEF with evolving treatment implications. Circulation 133(3), 245-247 (2015).

9 Jacobsen H, Ozmen L, Caruso A et al. Combined treatment with a bace inhibitor and anti-A $\beta$ antibody gantenerumab enhances amyloid reduction in APPLondon mice. J. Neurosci. 34, 11621-11630 (2014).

10 Seo SW, Ayakta N, Grinberg LT et al. Regional correlations between $\left[{ }^{11} \mathrm{C}\right]$ PIB PET and post-mortem burden of amyloidbeta pathology in a diverse neuropathological cohort. NeuroImage Clin. 13, 130-137 (2017).
- Shows the utility of Pittsburgh compound B as gold standard for in vivo imaging of amyloidosis-related disease.

11 Higuchi M, Iwata N, Matsuba Y et al. 19F and 1H MRI detection of amyloid $\beta$ plaques in vivo. Nat. Neurosci. 8 , 527-533 (2005).

12 Rapezzi C, Quarta CC, Guidalotti PL et al. Role of 99mTcDPD scintigraphy in diagnosis and prognosis of hereditary transthyretin-related cardiac amyloidosis. J. Am. Coll. Cardiol. Img. 4, 659-670 (2011).

13 Yoshimura M, Ono M, Watanabe H, Kimura H, Saji H. Feasibility of amylin imaging in pancreatic islets with $\beta$-amyloid imaging probes. Sci. Rep. 4, 6155 (2014).

14 Knight MJ, McCann B, Kauppinen RA, Coulthard EJ. Magnetic resonance imaging to detect early molecular and cellular changes in Alzheimer's disease. Front. Aging Neurosci. 8, 139 (2016).

15 Moses WW. Fundamental limits of spatial resolution in PET. Nucl. Instrum. Methods Phys. Res. A 648(Suppl. 1), S236-S240 (2011).

16 Martí-Bonmatí L, Sopena R, Bartumeus P, Sopena P. Multimodality imaging techniques. Contrast Media Mol. Imaging 5, 180-189 (2010).

- Significance on combination of various imaging techniques in diagnosis, adding functional and structural data.

17 Huang WY, Davis J. Multimodality and nanoparticles in medical imaging. Dalton Trans. 40, 6087-6103 (2011).

18 Lux F, Mignot A, Mowat P et al. Ultrasmall rigid particles as multimodal probes for medical applications. Angew. Chem. Int. Ed. Engl. 50, 12299-12303 (2011).

19 LeDuc G, Roux S, Paruta-Tuarez A et al. Advantages of gadolinium based ultrasmall nanoparticles vs molecular gadolinium chelates for radiotherapy guided by MRI for glioma treatment. Cancer Nanotechnol. 5, 4 (2014).

20 Sancey L, Lux F, Kotb S et al. The use of theranostic gadolinium-based nanoprobes to improve radiotherapy efficacy. BJR 87, 20140134 (2014). 
-. Review and description of imaging properties of versatile AGuIX nanoparticles.

21 Truillet C, Bouziotis P, Tsoukalas C et al. Ultrasmall particles for Gd-MRI and 68Ga-PET dual imaging. Contrast Media Mol. Imaging 10, 309-319 (2015).

22 Bianchi A, Moncelet D, Lux F et al. Orotracheal administration of contrast agents: a new protocol for brain tumor targeting. NMR Biomed. 28, 738-746 (2015).

23 Pansieri J, Plissonneau M, Heinrich-Balard L et al. Gdnanoparticles functionalization with specific peptides for ß-amyloid plaques targeting. J. Nanobiotechnol. 14, 60 (2016).

24 Biancalana M, Koide S. Molecular mechanism of Thioflavin-T binding to amyloid fibrils. Biochim. Biophys. Acta 1804, 1405-1412 (2010).

$25 \mathrm{Ng}$ S, Villemagne VL, Berlangieri S et al. Visual assessment versus quantitative assessment of 11C-PIB PET and 18F-FDG PET for detection of Alzheimer's disease. J. Nucl. Med. 48, 547-552 (2007).

26 Maezawa I, Hong HS, Liu R et al. Congo red and thioflavin- $\mathrm{T}$ analogs detect $\mathrm{A} \beta$ oligomers. J. Neurochem. 104, 457-468 (2008).

27 Ni R, Gillberg PG, Bergfors A, Marutle A, Nordberg A. Amyloid tracers detect multiple binding sites in Alzheimer's disease brain tissue. Brain 136, 2217-2227 (2013).

28 Lux F, Sancey L, Bianchi A et al. Gadolinium-based nanoparticles for theranostic MRI-radiosensitization. Nanomedicine 10, 1801-1815 (2015).

29 Wang Y, Fan Z, Shao L et al. Nanobody-derived nanobiotechnology tool kits for diverse biomedical and biotechnology applications. Int. J. Nanomedicine 11, 3287-3303 (2016).

30 Sillerud LO, Solberg NO, Chamberlain R et al. SPIONenhanced magnetic resonance imaging of Alzheimer's disease plaques in A $\beta$ PP/PS-1 transgenic mouse brain. J. Alzheimers Dis. 34, 349-365 (2013).

31 Habicht G, haupt C, Friedrich RP et al. Directed selection of a conformational antibody domain that prevents mature amyloid fibril formation by stabilizing A $\beta$ protofibrils. Proc. Natl Acad. Sci. USA 104, 19232-19237 (2007).

32 Haupt C, Bereza M, Kumar ST et al. Pattern recognition with a fibril-specific antibody fragment reveals the surface variability of natural amyloid fibrils. J. Mol. Biol. 408, 529-540 (2011).

- High-affinity nanobody B10AP for amyloid fibrils, implicated in various diseases.

33 Martins AF, Morfin JF, Kubickova A et al. PiB-conjugated, metal-based imaging probes: multimodal approaches for the visualization of $\beta$-amyloid plaques. ACS Med. Chem. Lett. 4, 436-440 (2013).

34 Cao P, Tu LH, Abedini A et al. Sensitivity of amyloid formation by human islet amyloid polypeptide to mutations at residue 20. J. Mol. Biol. 421, 282-295 (2012).
35 Borchelt DR, Davis J, Fischer $\mathrm{M}$ et al. A vector for expressing foreign genes in the brains and hearts of transgenic mice. Genet. Anal. 13, 159-163 (1996).

36 Borchelt DR, Thinakaran G, Eckman CB et al. Familial Alzheimer's disease - linked Presenilin 1 variants elevate A $\beta 1-42 / 1-40$ ratio in vitro and in vivo. Neuron 17 , 1005-1013 (1996).

37 Janson J, Soeller WC, Roche PC et al. Spontaneous diabetes mellitus in transgenic mice expressing human islet amyloid polypeptide. Proc. Natl Acad. Sci. USA 93, 7283-7288 (1996).

38 Kohno K, Palha JA, Miyakawa K et al. Analysis of amyloid deposition in a transgenic mouse model of homozygous familial amyloidotic polyneuropathy. Am. J. Pathol. 150, 1497-1508 (1997).

39 Palha JA, Episkopou V, Maeda S, Shimada K, Gottesman ME, Saraiva MJM. Thyroid hormone metabolism in a transthyretin-null mouse strain. J. Biol. Chem. 269, 33135-33139 (1994).

40 Maskevich AA, Stsiapura VI, Kuzmitsky VA et al. Spectral properties of thioflavin $\mathrm{T}$ in solvents with different dielectric properties and in a fibril-incorporated form. J. Proteome Res. 6, 1392-1401 (2007).

41 Rodina NP, Sulatsky MI, Sulatskaya AI, Kuznetsova IM, Uversky VN, Turoverov KK. Photophysical properties of fluorescent probe Thioflavin $\mathrm{T}$ in crowded milieu. J. Spectrosc. 2017, 2365746 (2017).

42 Petiet A, Santin M, Bertrand A et al. Gadoliniumstaining reveals amyloid plaques in the brain of Alzheimer's transgenic mice. Neurobiol. Aging 33, 1533-1544 (2012).

43 Terazaki H, Ando Y, Fernandes R, Yamamura KI, Maeda S, Saraiva MJM. Immunization in familial amyloidotic polyneuropathy: counteracting deposition by immunization with a Y78F TTR mutant. Lab. Invest. 86, 23-31 (2005).

44 Lia T, Vandesquille M, Koukoulik F et al. Camelid singledomain antibodies: a versatile tool for in vivo imaging of extracellular and intracellular brain targets. J Control. Release 243, 1-10 (2016).

45 Xiong N, Dong XY, Zheng J, Liu FF, Sun Y. Design of LVFFARK and LVFFARK-functionalized nanoparticles for inhibiting amyloid $\beta$-protein fibrillation and cytotoxicity. ACS Appl. Mater. Interfaces 7, 5650-5662 (2015).

46 Moss MA, Nichols MR, Reed DK, Hoh JH, Rosenberry TL. The peptide KLVFF-K(6) promotes beta-amyloid(1-40) protofibril growth by association but does not alter protofibril effects on cellular reduction of 3-(4,5-dimethylthiazol2-yl)-2,5-diphenyltetrazolium bromide (MTT). Mol. Pharmacol. 64, 1160-1168 (2003).

47 Sancey L, Kotb B, Truillet C et al. Long-term in vivo clearance of gadolinium-based AGuIX nanoparticles and their biocompatibility after systemic injection. ACS Nano 9, 2477-2488 (2015). 